\title{
Chemists crack complex compound
}

\section{Naturalistic approach vindicated as sponge molecule yields to synthesis in the lab.}

One of the most daunting challenges for synthetic chemists has finally been conquered. The effort to make palau'amine in the lab sparked heated competition for more than a decade between leading researchers, even though it may have little potential as a drug.

The yield of the 25-step synthesis, which was led by Phil Baran at the Scripps Research Institute in La Jolla, California, was just $0.015 \%$ : fewer than 2 in every 10,000 molecules of starting material made it through to the final product.

"Palau'amine is the pinnacle of technical difficulty," says organic chemist Patrick Harran of the University of California, Los Angeles, who has been trying to make the compound since 2002. "Phil and his students have set a standard against which all future work in the area will be judged."

But the synthesis, published last week in Angewandte Chemie ${ }^{1}$, is more than a technical achievement. The procedure demonstrates the effectiveness of a set of guiding principles for efficient organic synthesis that was articulated by Baran's group several years ago and is now gaining adherents for its focus on brevity and simplicity.

Palau'amine was isolated from the sponge Stylotella agminata, which is found in the waters around the Republic of Palau in the

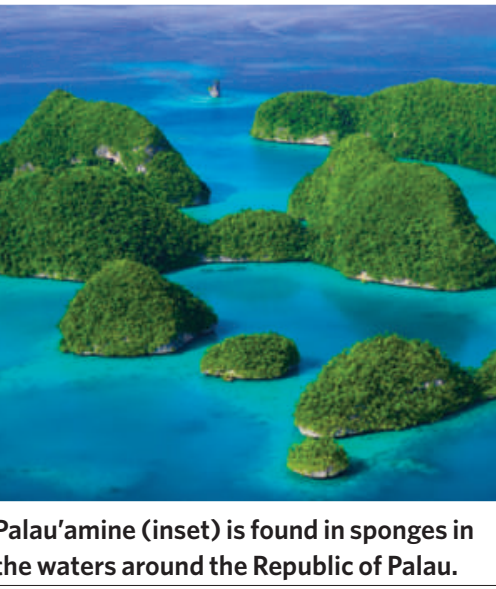

western Pacific Ocean. First reported in 1993 (ref. 2), it is part of a family of compounds known as pyrrole-imidazole alkaloids, which may help to deter fish from snacking on the sponge or prevent microbes from taking up residence. The molecule has antitumour, antibacterial and antifungal activity at levels that are "OK, but not fantastic", says Matthias Köck, a marine natural-products chemist at the Alfred Wegener Institute for Polar and Marine Research in Bremerhaven, Germany.

Thus, the main attraction in synthesizing the molecule is not its potential as a wonder drug, but the sheer challenge of making something so complex. The structure of palau'amine is crowded with spurs and joints in unusual places, and littered with nitrogen atoms that lie in wait to disrupt the chemical reactions used to stitch the compound $\frac{}{\infty}$ together. At the molecule's heart lies a unique configuration of rings - two circles made up of carbon atoms and a nitrogen atom that are fused in a contorted configuration.

For more than a decade, chemists assumed that the rings were twisted into a bowl-like shape. But in 2004, Baran hypothesized that all the members of palau'amine's molecular family could be constructed with the same general strategy - implying that the accepted structure of palau'amine was wrong.

In 2007 , his prediction was vindicated by three teams that independently worked out its true structure. Köck, who led the most detailed study, recalls that at first "almost no one believed us. Nearly everyone we spoke to thought we were misguided." But synthetic chemists soon switched their focus to the revised target. "Many groups had been chasing the wrong structure for years," says Köck.

Baran's synthesis adheres to a set of synthesis guidelines ${ }^{3}$ that aims to exploit the target molecule's inherent reactivity and stay as close as possible to the way it is made in

\section{Israeli government advisers threaten walkout}

\section{A rift in Israel's science} establishment is threatening the country's long-term planning of civilian science. All 15 members of its National Council for Research and Development, now subordinate to the ministry of science, are poised to resign this month unless the council is given independent budgetary and administrative standing.

"If the council ceases to exist, we won't see any effects in the short term," comments Meir Zadok, director of the Israel Academy of Sciences and Humanities in Jerusalem. “But its responsibility is to aggregate information about research and development throughout the country and to look ten years ahead to see where the government needs to be involved."

In recent years the council has lent its support to efforts to increase research and university funding. Members have appeared in public forums and in the Knesset, Israel's parliament, to lobby for such funding.

The group was established in its current form in 2004 as part of the Israel Academy of Sciences and Humanities. In 2007, under the previous government, the council was transferred to the ministry of science when a science minister wanted to expand the ministry's responsibilities.

"The council's budget currently comes through the ministry of science, and it requires the approval of the ministry's officials for everything it does," says Meir Sheetrit, chairman of the science and technology committee in the Knesset. Council members chafed at being subject to the ministry's whims, and complained that their budget was being cut in favour of other areas under the ministry's purview, which included - until last spring - culture and sport. In response, Sheetrit held hearings and drafted legislation to change the council's status to that of a government-run corporation. "Everywhere else in the world," he says, "national research councils are independent, with separate budgets, to ensure their objectivity."

But the government opposed the bill and, in mid-December, the Knesset rejected the legislation. As a result, council members plan to submit their resignations en masse in the next few weeks.

Council director Rony Dayan blames Daniel Hershkowitz, the 
nature, explains team member Ian Seiple. This includes using cascade reactions that can form many new chemical bonds in a single step, and avoiding the use of protecting groups to shield fragile parts of a molecule during synthesis because they increase the cost and complexity of the process.

Although none of the guidelines is new, applying them all within the same synthesis has become a hallmark of Baran's work. His goal is to prove that new drugs do not have to be built from the relatively limited pool of molecular motifs used by pharmaceutical companies.

The efforts to synthesize palau'amine have forced chemists to develop new reactions and techniques for assembling complicated molecules. Part of Baran's synthesis relies on a silver-based reagent, for example, that his lab invented to gently oxidize the half-built palau'amine molecule without disrupting its nitrogen atoms. That reagent is already being used by a pharmaceutical company to make a range of drug candidates, says Baran.

In the near future, he hopes to make grams of the compound instead of the few milligrams he has so far achieved, and to tweak his synthesis so that just one of the two possible mirrorimage forms of the compound is produced. His team already has a working route that cuts ten steps from the beginning of the process. "For us, the story has just begun," Baran says. Mark Peplow

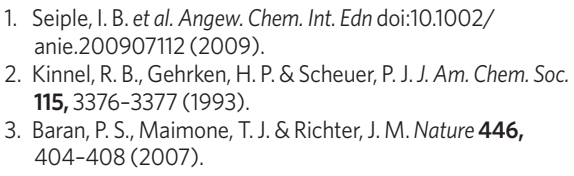

country's science minister, for torpedoing the legislation. "The officials in his ministry warned him that his small ministry would have trouble justifying its existence if it lost authority over the council," says Dayan.

Hershkowitz rejects that charge and says he supports independent status for the council. "The law that was submitted wasn't appropriate, however," he told Nature. "My goal is that the research council operates with independence, but there needs to be oversight to ensure proper management."

He declined to comment on how he would react to a full council resignation.

Hershkowitz says that he plans to draft legislation in line with his goals of continued ministry oversight. Sheetrit has already reintroduced his own bill, and plans to continue to push for its passage.

Haim Watzman

\section{Kepler finds its first planets}

\section{WASHINGTON DC}

Stars hum and throb, and the vibrations of this cosmic music could aid the NASA satellite Kepler in its goal of finding an Earth-like extrasolar planet.

On 4 January at a meeting of the American Astronomical Society in Washington DC, the Kepler team announced that it had identified five new planets. These are the first to be found by the 1-metre telescope, which stares continuously at one swathe of sky and looks for the dimming as a planet crosses a star and blocks some of its light. Hundreds more planet candidates await confirmation as the telescope gathers more data. These include some that orbit stars bright enough for their characteristic 'asteroseismology' vibrations to be detected, says Ronald Gilliland, a Kepler team member at the Space Telescope Science Institute in Baltimore, Maryland.

A precise understanding of these vibrations could allow astronomers to separate Earth-sized planets into two groups: those that are rocky and those that are watery, says Dimitar Sasselov, a co-investigator on the Kepler science team and an astronomer at Harvard University in Cambridge, Massachusetts. "It makes all the difference."

Because the core of an older star vibrates differently from that of a younger one, asteroseismology measurements can allow a precise determination of the age of the star system (and thus the planet). The data can also lead to a better estimate of the star's size - which in turn leads to more precision in the planet size. Gilliland says the extra precision could, when combined with ground-based measurements, help to determine the density of exoplanets as much as $50 \%$ better than before. Sasselov says that will be just enough of an improvement to discern the difference between a rocky planet like Earth, like the recently discovered GJ $1214 \mathrm{~b}$, which is probably at least $50 \%$ water (D. Charbonneau et al. Nature 462 . 891-894; 2009).

It is still early days for Kepler, which launched on 6 March 2009 from Cape Canaveral, Florida. The first five planets were discovered with just the first six weeks' science data, and they cross their parent stars repeatedly in short period orbits of a few days. Four are bigger than Jupiter - the largest planet in our Solar System - and one is about the size of Neptune.

Orbs more like Earth will be seen as the team shifts its attention to smaller planets in longer period orbits.

But Kepler only measures size. To understand density the team needs to measure mass as well, which comes from follow-up observations by ground-based astronomers. Sasselov says that even the giant 10-metre Keck telescopes in Hawaii lack an instrument sensitive enough to confirm an Earth-like planet if Kepler saw it. He is building a new instrument that he hopes to have installed on the 4.2-metre William Herschel Telescope in the Canary Islands by 2011 or 2012 - about the time when Kepler should have Earth-analogue candidates to check. Eric Hand which is $0.06 \%$ water, and a water world

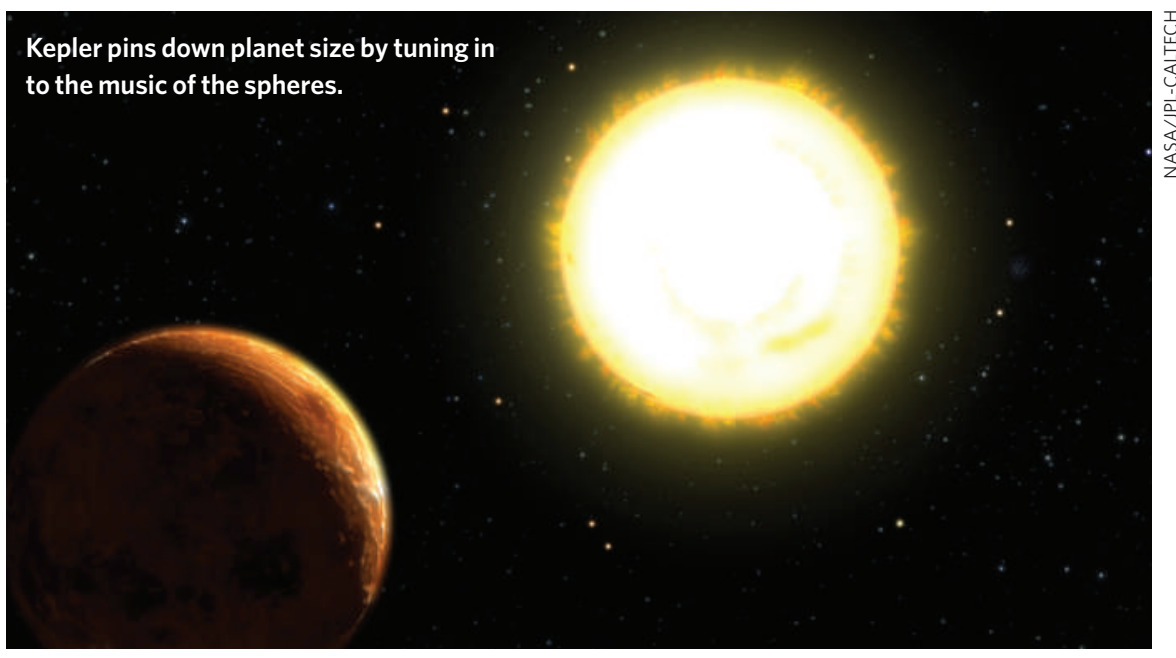

